\title{
El importante rol de las empresas en situaciones de crisis sanitaria: ¿un Plan de Prevención Corporativo?
}

\section{The Important Role of Companies in Situations of Health Crisis: A Corporate Prevention Plan?}

\section{MaRIe-Christine Fuchs ${ }^{1}$ y Miguel Barboza LóPeZ ${ }^{2}$}

Resumen: El Covid-19 ha puesto en jaque a diversos actores de nuestra sociedad, entre ellos las empresas. El rol empresarial, dentro del marco de una debida diligencia en su actuar, en tiempos de pandemia, es trascendental no solo para asegurar los derechos humanos de sus trabajadores o entorno cercano, sino también para el bienestar común de la sociedad. Frente a esta situación, el presente artículo propone la necesidad de contar con un Plan de Prevención Corporativo ante emergencias sanitarias bajo siete frentes claves, a fin de que las empresas puedan anticiparse a los efectos que puede tener una pandemia $u$ otra emergencia en sus actividades.

Palabras claves: Emergencia sanitaria, empresas, Plan de Prevención Corporativo, debida diligencia

Abstract: Covid-19 has challenged various actors in our society, including companies. The role of businesses during the pandemic, within the framework of due diligence, is essential not only to ensure

\footnotetext{
${ }^{1}$ Directora del Programa Estado de Derecho para Latinoamérica de la Fundación Konrad Adenauer.

${ }^{2}$ Coordinador de Proyectos del Programa Estado de Derecho para Latinoamérica de la Fundación Konrad Adenuaer.
} 
the human rights of its workers and those close to them, but also for the common welfare of society. Faced with this situation, this article advances the need to come up with a Corporate Prevention Plan for health emergencies under seven key fronts, so that companies can anticipate the effects that a pandemic or other emergency may have on their activities.

Keywords: Health emergency, Business, Corporate Prevention Plan, Due diligence

Recibido: 6.10.2020 Aceptado: 26.11.2020

Sumario

1. Generalidades

2. Reacción empresarial frente al Covid-19

3. Un Plan de Prevención Corporativo ante una crisis sanitaria

a. La actividad directa de la empresa, sus operaciones y sus impactos

b. El costo de la prevención y la debida diligencia frente a una situación de crisis sanitaria

c. La seguridad y salud en el trabajo de los trabajadores y las trabajadoras y de aquellos que operan en la cadena de valor tanto en tiempos de normalidad como en tiempos de crisis sanitaria

d. Los impactos en los derechos laborales de los trabajadores, independientemente de los derivados de la seguridad y salud en el trabajo

e. Tomar en consideración a los consumidores y las consumidoras

f. Poblaciones en especial situación de vulnerabilidad: mujeres, niños, personas adultas mayores, y pueblos indígenas 


\section{g. Responsabilidad social empresarial}

\section{4. ¿Un Plan de Prevención Corporativo puede manejarse sin una política sólida nacional en empresas y derechos humanos?}

\section{Conclusión}

\section{Generalidades}

La pandemia del Covid-19, reconocida así por la Organización Mundial de la Salud (OMS, 2020) ha cobrado la vida de miles de personas alrededor del mundo y afectado gravemente la actividad económica mundial (ONU, 2020c). La pandemia viene poniendo a prueba no solo la capacidad de respuesta de los Estados para hacer frente a esta grave crisis sanitaria, sino también la de los demás actores de la sociedad, como las empresas.

El riesgo de propagación de infecciones (que posteriormente puede generar una pandemia) y su manejo no es un tema aislado del actuar de los Estados, las empresas y la sociedad civil. Por tanto, se requiere de un fuerte compromiso que esté cohesionado no solo en una política estatal en materia de derechos humanos y empresas (coherencia política y normativa), sino también en la voluntad particular tanto corporativa como de la ciudadanía en general.

Las empresas, desde sus diferentes dimensiones corporativas, pueden apoyar positivamente el desarrollo del flujo económico de nuestros países a través de la generación de empleos, la creación de nuevas iniciativas de negocio, el abastecimiento de productos y alimentos, el impulso de servicios sociales indispensables (transporte, educación y salud), la reducción de la pobreza, entre otros. No obstante, según la OMS, los impactos negativos que sus actividades podrían tener directa o indirectamente en el incremento del riesgo de propagación de infecciones también son altos y agravarían una situación de pandemia. Entre estos impactos negativos podemos señalar la contribución al fenómeno migratorio, el cambio climático 
(BBC Mundo, 2019), a través de la producción de gases efecto invernadero en actividades extractivas y de inversión, las evasiones tributarias que fomentan la pobreza y la desigualdad, entre otros (OMS, 2020).

Frente a ello, en esta nueva realidad que estamos enfrentado con el Covid-19 resulta pertinente y necesario que las empresas desarrollen sus actividades bajo una óptica clara de debida diligencia y de prevención. Una manera de lograrlo es con la formulación e implementación de un Plan de Prevención Corporativo (PPC o Plan), el cual es un instrumento propositivo de gestión empresarial que recoge buenas prácticas de debida diligencia para prevenir o enfrentar riesgos que se pueden generar en el desarrollo de sus actividades durante una pandemia u otras situaciones de emergencia, evitando así violaciones a derechos humanos, que pondrían en riesgo su interés legítimo de generar ganancias. Cabe precisar que en la actualidad, al ser un tema reciente y que recién se está planteando, no se han estructurado posiciones contrarias al desarrollo de un instrumento de gestión corporativa especializado como el propuesto.

En esta línea, el presente artículo abordará por qué un PPC es esencial dentro de la labor de toda empresa frente a una situación de crisis como el Covid-19, y qué elementos deberían tomarse en cuenta dentro de su elaboración. Abordaremos el tema en tres partes. La primera describirá muy brevemente cuál ha sido la reacción empresarial (positiva y negativa) frente al Covid-19 durante los primeros meses en que se enfrentó la pandemia en Latinoamérica, punto álgido para saber si las empresas estuvieron o no preparadas para enfrentarlo. La segunda explicará la necesidad de contar con un PPC y los siete frentes que este tiene que incorporar para su efectividad. Finalmente, el tercero enfatizará la necesidad de contar con una política estatal sólida que robustezca la implementación de un PPC por parte de las empresas.

En la actualidad no existen lineamientos claros respecto a cómo las empresas deben abordar un PPC y menos aún en una situación de 
pandemia. El presente texto busca brindar una herramienta innovadora, clara y puntual sobre los siete elementos esenciales que deberá contener este Plan. Este puede ayudar a prevenir y enfrentar diversas situaciones de riesgo, haciendo así que los instrumentos corporativos de gestión sean dinámicos y propositivos para buscar soluciones y alimentar las políticas públicas sobre prevención y sobre empresas y derechos humanos.

\section{Reacción empresarial frente al Covid-19}

El impacto del Covid-19 en las empresas, al igual que otras situaciones de emergencia, sin duda es significativo y esto también pone a prueba su actuar en cuanto a cómo enfrentar esta pandemia y sus efectos posteriores. Al respecto, la declaración conjunta que han realizado la Organización Internacional del Trabajo, la Comisión Interamericana de Derechos Humanos y la Organización para la Cooperación y el Desarrollo Económicos (OCDE), entre otras organizaciones, ha enfatizado que los "factores como la rápida disminución de la actividad económica de los principales socios comerciales, la interrupción de las cadenas de valor mundiales, la caída de los precios de los productos básicos, el aumento de la aversión al riesgo de los inversores, la reducción de la demanda de mano de obra, especialmente en los sectores del turismo, la manufactura y el comercio minorista, y la caída de las remesas" (Organización Internacional del Trabajo et al, 2020) tendrán un profundo impacto en la actividad empresarial de América Latina y el Caribe.

Pese a este panorama devastador para la economía de los empresarios y las empresarias y la economía nacional y mundial, observamos con optimismo cómo algunas empresas han implementado de inmediato ciertas políticas corporativas propositivas para enfrentar el Covid-19. Por mencionar algunas de estas empresas, tenemos a Pastelería San Antonio, ETB y Movistar, que han implementado diferentes medidas - tanto directamente por 
iniciativa de las empresas, como por disposición de los Estadostales como prórrogas al pago de obligaciones financieras ${ }^{3}$ (pese a que actualmente hay una controversia sobre el pago de intereses sobre cuotas no pagadas), facilidades para el uso de las telecomunicaciones, ${ }^{4}$ la continuidad en la producción de bienes de primera necesidad, y la estabilidad laboral de sus trabajadores y trabajadoras. ${ }^{5}$ No obstante, estas medidas se dieron sin existir un plan previo para enfrentar situaciones de emergencia sanitaria, y sin avizorar las medidas de seguridad y salud tanto en el trabajo como en el ámbito de la producción en general (esta última, además, afecta a la comunidad global).

Esta sinergia positiva, no obstante, no ha sido seguida por todo el sector empresarial. No todas las empresas han reaccionado de la misma manera acorde a sus deberes de debida diligencia $y$ prevención. Algunos simplemente no han podido, porque debido a la pandemia se han declarado en bancarrota. Hemos sido testigos de cómo algunos de estos actores han desconocido los derechos laborales de sus trabajadores y han impulsado la continuidad de sus actividades sin implementar ningún mecanismo de seguridad, así

\footnotetext{
${ }^{3}$ En Colombia y otros países, diversos bancos, como el BBVA, han otorgado un periodo de gracia de hasta 6 meses para el pago de ciertas de obligaciones de crédito para aquellos clientes que se encontraban al día al cierre de febrero, incluyendo capital, intereses y otros conceptos: BBVA. (2020). Programa de acompañamiento BBVA. https://www.bbva.com.co/personas/promocion/coronavirus.html\#credito-personal.

${ }^{4}$ En Colombia, Asomovil y Andesco que cuentan como empresas asociadas a Claro, Movistar, DirecTV, ETB y EMCALI anunciaron han anunciado que brindarán minutos gratis para llamadas celulares y un paquete de datos gratuitos a todos sus clientes, así como el aumento en la velocidad el servicio de internet durante la cuarentena: El Tiempo. (2020). Minutos y mensajes de texto gratis durante la cuarentena por coronavirus.https://www.eltiempo.com/tecnosfera/novedades-tecnologia/minutosy-mensajes-de-texto-gratis-durante-la-cuarentena-por-coronavirus-476112.

${ }^{5}$ En Perú, la Pastelería San Antonio cerró sus tiendas como una medida para evitar la propagación del coronavirus. Frente a ello, declaró que continuatá pagando los salarios y beneficios sociales a sus empleados y empleadas: Publimetro.2020. El ejemplar gesto de pastelería San Antonio ante cuarentena. https://www.publimetro.pe/nacional/2020/03/16/coronavirus-el-ejemplar-gesto-depasteleria-san-antonio-ante-cuarentena-noticia/.
} 
como la continuidad de actividades de alto riesgo (minería, hidrocarburos, hidroeléctricas, forestales, etc.). Esto ha tenido efectos devastadores para grupos en especial situación de vulnerabilidad y ha puesto en riesgo su salud, la de sus familias y la de la sociedad en general. ${ }^{6}$

En una situación de pandemia, en donde la economía se ve gravemente afectada por el bajo consumo de bienes, es claro que la resistencia de los empresarios por continuar con sus actividades corporativas se ve seriamente afectada, limitando así su continuidad y con ella la garantía de determinadas obligaciones hacia consumidores, trabajadores, y la comunidad en general. No obstante, en este punto es donde se tiene que buscar un actuar corporativo propositivo a fin de evaluar una potencial situación de riesgo, elaborar un PPC, y proyectar su ejecución con eficiencia y eficacia.

\section{Un Plan de Prevención Corporativo ante una crisis sanitaria}

Los Principios Rectores sobre Empresas y Derechos Humanos establecen el deber que tienen las empresas tanto de abstenerse de infringir los derechos humanos de terceros como de hacer frente a las posibles consecuencias negativas sobre los derechos humanos de las actividades en las que tengan participación (OACNUDH, 2011,

\footnotetext{
${ }^{6}$ Por ejemplo, en Colombia, las mujeres Wayuu denunciaron que la compañía Cerrejón que realiza minería de carbón en la Guajira reanudó sus operaciones durante el Covid19 , poniendo en riesgo a la comunidad y aumentando su vulnerabilidad en un contexto de escacez de agua: Contagio Radio. 2020. Comunidades Wayúu acuden a la ONU para frenar la actividad del Cerrejón en medio de la pandemia. https://www.contagioradio.com/comunidades-wayuu-acuden-a-la-onu-para-frenarla-actividad-del-cerrejon-en-medio-de-la-pandemia/.

Por otro lado, MiningWatch Canadá reportó que en países como Brasil, Ecuador, México, Honduras, Panamá, Colombia y Chile, en donde se declaró la cuarentena sanitaria pero la minería se consideró como una actividad esencial que les permitió seguir operando, los casos de Covid subieron alarmantemente: Miningwatch Canadá. 2020. Al ritmo del COVID-19, la minería ataca pueblos íntegros e indígenas. https://miningwatch.ca/node/10452.
} 
principio 11). Esto implica tomar medidas adecuadas para prevenir, mitigar y reparar dichas consecuencias (OACNUDH, 2011, principio 15). Por ende, en una situación de pandemia, el deber de "abstención" no significa un "no hacer" por parte de las empresas, sino un "hacer" para evitar situaciones de riesgo y potenciales violaciones a los derechos humanos.

También debe precisarse que los trabajadores, y la sociedad en general, como actores no estatales, tienen un deber de solidaridad (Asamblea General de las Naciones Unidas, 2016b, párr. 38) para contribuir a la efectividad de las medidas de debida diligencia y prevención implementadas por las empresas. Este se ve manifestado a través del comportamiento diligente frente a las medidas adoptadas por las empresas para hacer frente a la pandemia.

La obligación positiva de "hacer" de las empresas se puede traducir en el desarrollo de un PPC que deberá estar incluido dentro de las políticas corporativas de las empresas sobre derechos humanos y tener un periodo de evaluación permanente de acuerdo a la coyuntura económica, social y política. Una cultura preventiva en materia de salud podría garantizar el respeto de los derechos de los trabajadores y trabajadoras, el personal involucrado en su cadena de valor, la comunidad en general, y el medio ambiente (Pacto Mundial, Red Española).

No obstante, para que las empresas incluyan este Plan dentro de sus políticas corporativas deberán contar con un gobierno corporativo sólido, con el fin de fortalecer su estructura interna (personal directivo y de gerencia) (Deloitte; OMS, 2020) y también sus relaciones con actores económicos externos. Esto les permitirá tomar medidas a corto y a largo plazo que sean efectivas, pues el gobierno corporativo implica el desarrollo de controles internos, un manejo de información dinámico, líneas de comunicación definidas (OCDE, 2020b) - particularmente con aquellas personas directamente afectadas por la crisis o sus efectos posteriores-, y un "adoctrinamiento conjunto" por parte del personal directivo y 
gerencial de la empresa, que a fin de cuentas tomará y ejecutará las decisiones frente a la crisis sanitaria.

La elaboración de un PPC integral sin duda tendrá sus complejidades. Una de estas grandes complejidades es el rastreo de todos los actores económicos involucrados en la actividad empresarial, es decir, los que integran la cadena de suministro de una empresa. No obstante, las empresas tienen la posibilidad de "liberar" o hacer un disclosure de esta información, con el objetivo de construir cuál es su cadena de suministro y comprender qué vulnerabilidades en tiempos de pandemia también se pueden fomentar desde estas aristas.

Tomando en cuenta estas generalidades, el Plan deberá reconocer que las situaciones de emergencia sanitaria potencialmente pueden presentarse, independientemente de si sus actividades contribuyen o no a ella. Para la efectividad del Plan, este puede trabajarse desde siete frentes teniendo en cuenta el grado de riesgo a la salud de las personas que circulan dentro de la cadena de valor de la empresa.

\section{a. La actividad directa de la empresa, sus operaciones y sus impactos}

Las actividades directas de la empresa las podemos clasificar en dos tipos. La primera está relacionada con la identificación de actividades propias y bajo su control directo, incluyendo su cadena de valor, que contribuyan al desarrollo de una posible y eventual crisis sanitaria, o a agravar una ya existente, como el Covid-19 (actividades corporativas de origen). La segunda está relacionada a aquellas actividades que fomentan una conducta empresarial poco diligente en medio de la crisis sanitaria, pese a que sus actividades directa o indirectamente no contribuyan al desarrollo de una crisis sanitaria o agraven una ya existente (actividades corporativas derivadas).

Las actividades corporativas de origen, entonces, serían aquellas identificadas por la OMS, como la contribución al cambio climático, 
la escasez de recursos de primera necesidad, la contaminación medioambiental, entre otras, que tienen consecuencias directas en la propagación o generación de una pandemia (CIDH, 2019). La OCDE, en la misma línea que la OMS, resaltó que "el cambio climático, la contaminación de los recursos hídricos y los factores desencadenantes de la pérdida de la biodiversidad, como son la deforestación y el comercio ilícito de especies silvestres, pueden aumentar el riesgo de nuevas pandemias", de igual modo que la contaminación atmosférica y la falta de acceso al agua (OCDE, 2020a).

Por su parte, las actividades corporativas derivadas serían aquellas que en tiempos de pandemia generan — si bien no de manera voluntaria en todos los casos- el desempleo y el subempleo como una causa básica para la generación de pobreza y pobreza extrema (CIDH, 2019, párr. 239), la falta de educación cívica y sanitaria de la población en general, la corrupción como interrupción directa para el abastecimiento monetario y financiero necesario para cubrir bienes de primera necesidad y de urgencia pública, entre otros.

Ahora bien, estos dos tipos de actividades corporativas tienen que ser abordados por las empresas desde lo que llamaremos una "visión corporativa estructural e intersectorial". Las empresas no deben ser ajenas a analizar los efectos diferenciadores que tendrían sus políticas y actividades para grupos en situación de vulnerabilidad, como las mujeres, los niños y niñas, los adultos mayores, los pueblos indígenas, las personas en situación de discapacidad, entre otros. Estos efectos deben ser tomados en consideración se esté o no en una situación de pandemia.

Las empresas, tomando en cuenta lo anterior, tienen la tarea de hacer un "rastreo general" sobre qué actividades o subactividades que desarrollan podrían fomentar o acrecentar una situación de riesgo ya identificada, como el Covid-19, o sin necesariamente pasar por una crisis sanitaria, podrían eventualmente contribuir a generarla.

Entonces, este rol de identificación no debe ser visto únicamente dentro de la lupa interna de la empresa, es decir, dentro de su 
“jurisdicción corporativa" - por ejemplo, exclusivamente dedicarse a identificar riesgos laborales de sus trabajadores dentro de sus instalaciones de trabajo-, sino desde una "jurisdicción global corporativa", que identifique aquellas acciones corporativas internas que pueden tener repercusiones externas que impacten en la sociedad en una situación de pandemia.

En la actualidad, hemos podido observar que estas tareas no han sido del todo abordadas dentro de diversos planes corporativos, salvo los temas laborales (seguridad y salud en el trabajo) que pertenecen únicamente a la "jurisdicción corporativa”. Por ejemplo, en el caso del Plan de la minera Quellaveco —operada por la transnacional Angloamerican en Perú—, una de las pocas empresas mineras que ha hecho público su "Plan para la vigilancia, prevención y control de Covid-19 en el trabajo” (Agloamerican Group, 2020), no se advierte qué medidas dentro de sus operaciones mineras se han tomado o se tomarán para reducir riesgos a comunidades aledañas o al medio ambiente. Ello no implica necesariamente que no se estén tomando; sin embargo, la transparencia de la información es vital en estos casos para generar confianza entre las comunidades y así hacerlas partícipes de ese Plan.

\section{b. El costo de la prevención y la debida diligencia frente a una situación de crisis sanitaria}

Las empresas para el diseño del Plan primero deben disponer de recursos económicos para su planificación, elaboración, revisión, pruebas de aplicación y eventual ejecución. Es decir, el Plan tiene una doble faceta: una preventiva que cubre el proceso de previo a la contingencia, es decir, meramente especulativa en caso de pandemia; y una faceta de debida diligencia propia en la aplicación del Plan.

En ese sentido, el Plan debe prever, en primer lugar, la elaboración de un estudio económico de costos, con el fin de proyectar si la empresa ante una eventual situación de crisis sanitaria estaría en condiciones de implementar los regulares o los mayores 
estándares de debida diligencia dentro de sus actividades, frente a la inevitable afectación económica que estas sufrirían. Si se determina que la empresa no estaría en condiciones económicas de cubrir este deber de debida diligencia, se tendrá que pensar en una salida provisoria para tratar de cubrir esta circunstancia, como ahorros sobre las utilidades o seguros alternos, entre otros.

En segundo lugar, en respuesta a los resultados de dicho estudio económico de costos, el Plan debe considerar un "proyecto de mitigación” que eventualmente pueda cubrir esos gastos mayores que demandaría la implementación de medidas más fuertes para cumplir con su deber de debida diligencia en tiempos de crisis. Estos proyectos de mitigación empresariales deberían ser acordados con los Estados y fiscalizados por estos, así como por otras empresas consultoras contables, financieras o especialistas en seguridad y salud en el trabajo, pues es inevitable que a nivel estatal se apoye al sector empresarial a hacer frente a una situación de crisis sanitaria.

\section{c. La seguridad y salud en el trabajo de los trabajadores y las trabajadoras y de aquellos que operan en la cadena de valor tanto en tiempos de normalidad como en tiempos de crisis sanitaria}

El convenio 155 de la OIT sobre seguridad y salud de los trabajadores resalta que los empleadores deben garantizar que "los lugares de trabajo, la maquinaria, el equipo y las operaciones y procesos que estén bajo su control son seguros y no entrañan riesgo alguno para la seguridad y la salud de los trabajadores" (OIT, 1981, artículo 16); asimismo, en cuanto a las cadenas de valor, precisa que "siempre que dos o más empresas desarrollen simultáneamente actividades en un mismo lugar de trabajo tendrán el deber de colaborar en la aplicación de las medidas previstas en el presente Convenio" (OIT, 1981, artículo 17). En este sentido, el Plan debe considerar que las actividades que desarrolla la empresa, así como aquellas que pertenecen a su cadena de valor, no deben exponer a los trabajadores a condiciones riesgosas de salud que puedan enfrentarse en una crisis sanitaria, reduciendo los riesgos al máximo posible. Por 
este motivo, en todo momento las empresas deben proporcionar a sus trabajadores el Equipo de Protección Personal (EPP) adecuado, y alinear las políticas de prevención de la empresa principal con aquellas de las empresas que integran su cadena de valor.

La OCDE ha precisado, y con mucho atino, que las empresas con prácticas sólidas en seguridad y salud en el trabajo pueden aprovechar estos procesos más rápidamente para responder a las amenazas a corto plazo (OCDE, 2020b), como el Covid-19.

Por otro lado, es importante analizar cuáles son las actividades necesariamente vitales y de primera necesidad, que merecen su continuidad en tiempos de crisis sanitaria y sobre las cuales se requerirá adoptar medidas especiales de seguridad y salud en el trabajo — como aquellas que cubren servicios públicos básicos o esenciales para el abastecimiento de la ciudadanía-, y cuáles no son esenciales y eventualmente deberían suspenderse.

El Alto Comisionado de las Naciones Unidas para los Refugiados ha precisado que estos servicios son los relacionados con alimentos, materiales de construcción de alojamientos, cocinas y combustible (ACNUR, 2012). Por su parte, los diversos países de Latinoamérica y de Europa que decretaron el aislamiento obligatorio han establecido como actividades de primera necesidad y que no quedan suspendidas aquellas relacionadas con la distribución y producción de alimentos, los servicios de salud, provisión de energía eléctrica, agua y saneamiento, servicios financieros y bancarios, provisión de combustible, distribución y producción de medicinas y artículos de higiene, y servicios de telefonía e internet (Fundación Konrad Adenauer, 2020). Si bien no existe una lista cerrada de servicios de primera necesidad que no pueden ser suspendidos, es importante considerar los antes mencionados, pues son básicos en momentos de crisis sanitaria. 


\section{d. Los impactos en los derechos laborales de los trabajadores, independientemente de los derivados de la seguridad y salud en el trabajo}

El pasado 18 de marzo, la OIT emitió su informe sobre los impactos que el Covid-19 tendría para el derecho al trabajo. En dicho informe no solo se reconoce el grave impacto económico que han sufrido las empresas y la recesión económica mundial a la que entraremos, sino también la afectación al derecho al trabajo en tres frentes: la cantidad de trabajo (desempleo y subempleo), la calidad de trabajo (salarios y acceso a servicios de la seguridad social) y la afectación a los grupos más vulnerables (OIT, 2020).

Frente a esta situación —que es muy compleja y sobre la cual no pretendemos hacer un abordaje profundo, ya que se requeriría analizar los pronósticos económicos y las realidades nacionales y regionales para poder llegar a consensos-, se propone que dentro del Plan se puedan dialogar soluciones o salidas laborales con los trabajadores frente a situaciones de crisis sanitaria.

Las salidas laborales en crisis sanitaria deben ser resultado de una participación activa de los trabajadores, no solo de la empresa, sino también de la cadena de valor, a fin de generar bienestar común y pervivencia de la actividad empresarial en su conjunto. La labor de interactuar con las cadenas de valor es muy importante, sobre todo porque haciéndolo se puede llegar a conocer verdaderamente cuál es el alcance empresarial de una determinada actividad económica. Al respecto, la OCDE indica que tomar decisiones en conjunto con las partes interesadas "ayudará a identificar soluciones viables a corto y largo plazo, para abordar las presiones de reducción de costos, incluida las soluciones en torno a los recortes salariales o la demora en los pagos salariales, al tiempo que permiten un inicio más rápido de las operaciones durante el periodo de recuperación" (OCDE, 2020b). En este sentido:

- En primer lugar, el Plan debe contemplar que en un escenario de crisis sanitaria no es posible dar por terminada la relación laboral 
de un trabajador por motivos de enfermedad, tal como lo ha establecido el artículo 6 del Convenio 158 de la OIT sobre la terminación de la relación de trabajo. Claramente este Convenio hace referencia a los contratos a tiempo indeterminado, no obstante, en su artículo 2.2. deja abierta la puerta para que dichas disposiciones también sean aplicadas a cualquier contrato, independientemente de su naturaleza. Ahora bien, a nivel corporativo, en el Plan se puede definir si la ausencia al trabajo por motivos de enfermedad también se puede extender para aquellas situaciones de aislamiento obligatorio por riesgo a adquirir dicha enfermedad en un contexto de pandemia.

- En segundo lugar, el Plan debe determinar cuáles actividades son esenciales y requieren continuidad en el lugar de trabajo frente a una situación de crisis sanitaria. Ello ayudará a determinar cuáles trabajadores pueden realizar teletrabajo y cuáles no, asegurando así las medidas de seguridad y salud en el trabajo, como anteriormente se advirtió. Estas medidas permiten asegurar de manera efectiva la productividad de la empresa sin afectar los derechos laborales de los trabajadores.

- En tercer lugar, el Plan debe prever si, por la naturaleza de sus actividades, la empresa podrá o no continuar operando durante la crisis sanitaria y después de ella. Esta situación se presenta en el caso de empresas que no se dedican a actividades de primera necesidad, como por ejemplo aquellas dedicadas al turismo o al comercio exterior (Asamblea General de la Organización de las Naciones Unidas, 2016a, párr.26.). Frente a esta situación, podrían analizarse - junto con los trabajadores, la empresa y el Estado correspondiente- mecanismos para estimular la economía de estas empresas y velar por la continuidad de sus actividades en la medida que sea posible.

- En cuarto lugar, el Plan puede establecer un fondo de emergencia empresarial para situaciones de crisis sanitaria, es decir, que permita estar preparados económicamente para asegurar los 
derechos laborales de los trabajadores por un periodo razonable, determinado de manera colectiva entre el empleador y los trabajadores, tomando en cuenta también la naturaleza de la actividad empresarial, la capacidad económica de la empresa, y la emergencia sanitaria. Entre estos derechos laborales encontramos el pago de los salarios, beneficios de la seguridad social, primas, entre otros. No obstante, las empresas no deben estar solas frente a estas medidas, sino que deben contar con el acompañamiento de los Estados, los cuales pueden ofrecer incentivos empresariales que se vean reflejados, por ejemplo, en la reducción de impuestos y mejores medidas de seguridad social (OIT, 2020, 9).

Para un manejo adecuado de dichos fondos, la empresa tendría que contar con un órgano de control corporativo contra la corrupción, el cual debería de existir en todo momento y no activarse únicamente frente a un escenario de crisis. Como ha señalado Transparencia Internacional en su reciente informe sobre corrupción y coronavirus, "la corrupción encuentra terreno fértil para prosperar en tiempos de crisis, sobre todo cuando las instituciones y los mecanismos de supervisión son débiles, y la confianza ciudadana es escasa... la identificación de estos riesgos de corrupción antes de que sucedan pueden ayudar a fortalecer nuestra respuesta global a la pandemia y ofrecer así los servicios de salud a quienes más lo necesitan" (Transparency International, 2020).

Las empresas no son ajenas a apoyar la lucha contra la corrupción, más aún en escenarios de pandemia, en donde la emergencia pasa de ser particular a ser generalizada. En este sentido, el órgano de control corporativo que aquí se propone debería contar con una fiscalización permanente por parte del Estado, y estar integrada por miembros de la dirección de la empresa y por representantes de los trabajadores de la empresa. 
- En quinto lugar, el Plan también deberá prever acuerdos de naturaleza colectiva con los trabajadores frente a situaciones de crisis sanitaria que se puedan reflejar en ajustes salariales, distribución de horas de trabajo, entre otros, teniendo en cuenta las condiciones económicas de la empresa y su objetivo válido de asegurar la supervivencia de esta.

\section{e. Tomar en consideración a los consumidores y las consumidoras}

Sin duda, cuando se habla de un Plan con estas características, se piensa en una dinámica que se active y opere únicamente a nivel interno corporativo (protección del empleado) y no siempre a nivel externo, es decir, frente a quienes como consumidores o no consumidores directos podrían llegar a ser afectados por las medidas tomadas para hacer frente a una situación de pandemia.

Los consumidores, sin duda, son el agente principal de un negocio. En este rubro tenemos a las empresas financieras y bancarias, los comercios de producción, distribución y comercialización de productos de consumo o servicios, así a como los Estados mismos, quienes son consumidores de productos derivados de la actividad privada, entre otros.

En la Declaración del Grupo de Trabajo de las Naciones Unidas sobre las Empresas y los Derechos Humanos, respecto al Covid-19, se manifestó la necesidad que las empresas tomen en consideración a los consumidores (ONU, 2020a). Pero entonces, ¿desde qué aristas el Plan debería regular la protección a los consumidores? Sugerimos que sea desde dos aristas. La primera relacionada a los consumidores de servicios en general, en donde en la medida de lo posible se deben habilitar los medios no presenciales para evitar el hacinamiento o la mayor presencia física, o, en todo caso, y siempre que sea un servicio indispensable, habilitar un estricto control de bioseguridad en el establecimiento comercial. La segunda es el aseguramiento de los bienes de primera necesidad a los consumidores. Sin duda, este es uno de los principales puntos de quiebre de la pandemia, pues se 
tiene que velar por la protección del consumidor, no solo respecto a que exista oferta suficiente de productos de primera necesidad, que se produzcan y se evite una eventual escasez ${ }^{7}$, sino que al ser productos de consumo directo y particularmente vulnerables, como los alimentos, bebidas, medicamentos, entre otros, los protocolos de sanidad en estos eslabones de la cadena alimentaria deberán ser cumplidos (CEPAL-FAO, 2020).

El Plan deberá recoger estas preocupaciones, no actuando de manera individual sino de manera colectiva, teniendo presente que, al ser una cadena de consumo, la interrupción de actividades de un actor económico puede frenar todo el proceso de producción y distribución de un servicio o producto. En este sentido, la CEPAL recoge las palabras de Mazzucato al señalar que "las empresas deberían empezar a comprometerse con la implementación de estrategias colectivas en favor de la comunidad, que ayuden a reducir los riesgos sistémicos" (Mazzucato, 2020) para el consumidor.

\section{f. Poblaciones en especial situación de vulnerabilidad: mujeres, niños, personas adultas mayores, y pueblos indígenas}

El Plan tiene que tener en cuenta los impactos diferenciados de la pandemia particularmente para los grupos vulnerables. Las medidas corporativas adoptadas no deben ser ajenas a esta realidad, particularmente cuando se trate de mujeres, niños y niñas, pueblos indígenas y personas adultas mayores, cuyos impactos, como se ha visto en los últimos reportes mundiales, son alarmantes.

\footnotetext{
${ }^{7}$ Por ejemplo, en Perú el pasado 21 de mayo de 2020 se anunció que el Gran Mercado Mayorista cerraría de manera parcial sus instalaciones por el Covid-19: https://www.peru-retail.com/lima-gran-mercado-mayorista-cerrara-de-

maneraparcial-por-el-coronavirus /. De la misma manera en Costa Rica el pasado 13 de mayo de 2020 se estimo que el $87 \%$ de las pymes cayeron o detuvieron su actividad como resultado de la crisis del COVID-19: https://www.elmundo.cr/economiaynegocios /87-de-las-pymes-cayeron-o-detuvieron-su-actividad-como-resultado-dela-crisis-delcovid-19/
} 
Con ello no queremos indicar que todas las actividades empresariales en tiempos de pandemia van a tener un impacto hacia estos grupos, pero sí que el Plan debe considerar esta posibilidad y actuar de una manera previsible a fin de no generar una mayor responsabilidad empresarial en caso de que se llegue a identificar el nexo entre la violación y el actuar corporativo.

Con relación a las mujeres, la OCDE ha señalado que corren un riesgo particular, pues integran una gran proporción de la fuerza laboral en sectores particularmente expuestos, como el comercio minorista de alimentos y la atención médica (OCDE, 2020c). Esta postura ha sido compartida por la Comisión Interamericana de Mujeres, que ha resaltado la necesidad de facilitar el teletrabajo y reconocido la alta carga familiar que tienen las mujeres (Comisión Interamericana de Mujeres, 2020). Desigualdad aún por corregir.

Por su parte, los niños y niñas y adolescentes, particularmente aquellos afectados por la pobreza y la violencia, tienen un grado de vulnerabilidad mayor al no contar con servicios básicos de salud, agua y saneamiento, nutrición y protección (UNICEF, 2020). La afectación a los niños y niñas y adolescentes podría parecer ajena a la actividad corporativa; no obstante, no lo es, pues los impactos pueden ser diversos de acuerdo con el rubro corporativo de la empresa. Por ejemplo, las medidas corporativas deben permitir una política de horario flexible para los padres que cumplen teletrabajo desde casa, de modo de prevenir la violencia intrafamiliar. De la misma manera, ante el escaso flujo económico producido por la pandemia, las empresas deben prevenir actos de servidumbre sexual de menores entre los actores económicos que actúan dentro de su cadena de valor (Pacto Global, Red Colombia 2020).

En cuanto a las personas adultas mayores, las cifras han arrojado que se tratan de una población de alto riesgo de contagio de Covid-19. Según información oficial al mes de julio de 2020, el número de personas adultas mayores que fallecieron en países como Cuba, México, Panamá, El Salvador, Guatemala y Costa Rica ha sido de 
18.548 (CEPAL, 2020). En este sentido, aquellas personas mayores que se encuentren laborando deberán contar con todas las facilidades necesarias, en la medida que sea posible, para hacer teletrabajo y no recibir actos de hostilidad por su situación de vulnerabilidad. Asimismo, las empresas deberán tener un control adecuado para identificar que sus actividades empresariales no generen un riesgo mayor a estas personas, especialmente en las actividades extractivas, de monocultivos, y de infraestructura.

Por último, los pueblos indígenas, especialmente aquellos en situación de aislamiento y contacto inicial (PIACI), son quizás uno de los grupos más afectados por la pandemia. El legajo de violaciones a estos pueblos, particularmente por el sector extractivo y de inversión, ha sido devastador, tanto para sus tierras, territorios y recursos naturales, como para su propia cosmovisión. Las empresas, por lo tanto, deberán fomentar el respeto de sus territorios, tierras y recursos naturales, evitando contaminarlos, a fin de no generar mayores impactos a su salud (FAO, 2020, recomendación 11).

\section{g. Responsabilidad social empresarial}

El deber de solidaridad (Asamblea General de la Organización de las Naciones Unidas, 2016b. párr. 38) anteriormente mencionado aplica también para las empresas en una situación de emergencia sanitaria como el Covid-19. Este deber precisa la necesidad de cooperación mutua ente los diversos actores de la sociedad, como las empresas, en situaciones donde el bien común se encuentra en riesgo.

Definitivamente, el deber de solidaridad en medio de la pandemia no debe ser un llamado a someter a las empresas a una carga "insoportable e inmanejable" de ayudar a mitigar los efectos del Covid-19 o encontrar una vacuna, pues las dimensiones económicas de las empresas son diversas y atienden a realidades diferentes. No obstante, en la medida de lo posible, el Plan debería contemplar una ayuda significativa, de acuerdo a la dimensión de la empresa y al 
impacto generado en la sociedad, para ayudar a los gobiernos centrales u organismos internacionales en su lucha.

Por ejemplo, grandes compañías multinacionales ${ }^{8}$ y otras empresas grandes y medianas de diversas partes del mundo han contribuido al Covid-19 Solidarity Response Fund (Empresa y Sociedad, 2020), llamado internacional que se dio desde el inicio de la pandemia hasta hace poco y en el cual la OMS requería de 31.000 millones de dólares para pruebas y vacunas contra el coronavirus (ONU, 2020b), sin dejar de lado las necesidades urgentes de diversos Estados para mediar los efectos del virus.

\section{4. ¿Un Plan de Prevención Corporativo puede manejarse sin una política sólida nacional en empresas y derechos humanos?}

Un PPC se fortalece cuando existe una política pública del Estado frente al tema de empresas, derechos humanos y manejo de crisis sanitarias mediante la cual se obtengan respuestas políticas a corto y largo plazo, que se basen en un enfoque integral de gobierno, en diálogo con las empresas, los trabajadores, y las personas afectadas (ONU, 2020a) y que busquen garantizar la prosperidad económica y el bienestar general a través de mejorar la productividad, reforzar la capacidad de resiliencia y descarbonizar la economía (OCDE, 2020a). La participación de estos actores en la formulación de políticas publicas preventivas no solo es deseable, sino imprescindible, pues de esta manera se logrará garantizar una sinergia en la forma de actuar ante situaciones de esta índole, en la cual debe primar la responsabilidad de los diferentes actores que interactúan en la sociedad para así reducir el impacto de la crisis.

\footnotetext{
${ }^{8}$ Twitter y Square, y Fortescue Metals donaron mas de mil millones de dólares para la lucha contra la Covid-19: MSN. 2020. Las 5 donaciones más grandes por el coronavirus: https://www.msn.com/es-co/dinero/finance/las-5-donaciones-m\%C3\%Als-grandespor-el-coronavirus/ar-BB13VRfq.
} 
Si bien las políticas públicas, materializadas en medidas económicas de apoyo a la crisis, tienen que responder de manera inmediata a una situación de grave emergencia, como lo es el Covid19, no deben de pensarse únicamente a corto plazo y con un enfoque restringido únicamente para reactivar la economía y proteger a las personas en riesgo en el ámbito del trabajo. En cambio, el enfoque debe ser pensado y planificado a largo plazo, a fin de que estas políticas públicas no se pierdan y tengan pervivencia en el tiempo, e impacten en el pleno respeto de los derechos humanos en el actuar empresarial.

Asimismo, las políticas públicas en materia de empresas y derechos humanos adoptadas en tiempo de crisis no deben dejar de lado aquellos vacíos políticos pendientes por resolver antes que el Covid-19 brote en nuestro continente. Por el contrario, es necesario que se robustezcan los esfuerzos políticos y se evalúen aquellas leyes o políticas que, como mencionamos anteriormente, podrían de una $u$ otra manera fomentar actividades corporativas que coadyuven al desarrollo de una pandemia o aceleren su brote en la sociedad.

En suma, en el campo de las políticas públicas, las empresas requieren de un marco jurídico sólido y coherente mediante el cual puedan armonizar su trabajo y encuentren el respaldo estatal necesario para hacerlo viable.

\section{Conclusión}

Frente a cualquier situación de crisis, sea sanitaria o no, resulta imprescindible que las empresas cuenten con un PPC. Esta poderosa herramienta de gestión corporativa contribuirá a que las empresas desarrollen sus actividades de una manera preventiva, respetando los derechos humanos en un marco de debida diligencia.

En el desarrollo de este corto artículo se ha observado, con algunos ejemplos, que algunas empresas han tenido un rol más director que otras en el manejo de sus actividades frente al Covid-19, 
pero no contaban - y quizás aún no cuenten - con un PPC frente a situaciones de riesgo sanitario. Esta herramienta de gestión corporativa es esencial y deberá ser abordada tomando en cuenta principalmente siete ejes: la actividad directa e indirecta de las empresas, el costo de la prevención y debida diligencia, la seguridad y salud en el trabajo, el impacto en los derechos laborales, la consideración a los consumidores de bienes y servicios, la población en especial situación de vulnerabilidad y la responsabilidad social corporativa.

En el proceso de elaboración y ejecución del Plan, las empresas necesitan manifestar su compromiso social, económico y político (OACNUDH, 2011, principio 16) frente a una situación de crisis. En este proceso, las empresas deben contar con un "asesoramiento especializado interno y/o externo” (OACNUDH, 2011, principio 16.b.) por parte de un equipo multidisciplinario tanto de profesionales de la salud, economía, leyes, entre otras ramas científicas. También es importante que las empresas no actúen solas, sino que sus planes estén acompañados de un fuerte compromiso de los Estados para hacerlos viables, teniendo en cuenta que las buenas prácticas corporativas pueden también fortalecer la legislación interna de un país cuando esta no sea suficientemente sólida.

Los esfuerzos corporativos por desarrollar este instrumento de gestión corporativa no solo mejorarán el manejo de futuras crisis sanitarias o de otra índole, sino que también marcarán una ruta clave para encaminar el actuar corporativo hacia la responsabilidad social de quienes dependen directamente de su actividad, como de aquellos que indirectamente resulten afectados. Este camino hacia la debida diligencia en el manejo de las crisis puede ir acompañado de incentivos corporativos, sean promovidos por los Estados, por ejemplo, mediante la reducción de impuestos; por cooperantes externos (instituciones financieras internacionales), a través de prestamos internacionales; o para aminorar la conflictividad social que podría generarse alrededor de las empresas por la pandemia e 
incluso posibles procesos judiciales o administrativos a nivel interno contra estas.

\section{Referencias}

Alto Comisionado de las Naciones Unidas para los Refugiados. 2012. Manual para situaciones de emergencia. https://www.acnur.org/fileadmin/Documentos/Publicaciones/ 2012/1643.pdf

Angloamerican. «Plan para la Vigilancia, prevención y control de COVID-19 en el trabajo», 14 de mayo de 2020. https://peru.angloamerican.com/ /media/Files/A/AngloAmerican-Group/Peru/documentos/4-plan-para-vigilancia-deCOVID19.pdf.

Asamblea General de la Organización de Naciones Unidas. 2016a. Informe del Grupo de Alto nivel sobre la Respuesta mundial a las Crisis Sanitarias: Proteger a la humanidad de futuras crisis sanitarias. Resolución. A/70/723.

Asamblea General de la Organización de Naciones Unidas. 2016b. Los derechos humanos y la solidaridad internacional: Informe de la experta independiente sobre los derchos humanos y la solidaridad intrnacional. Resolución A/71/280.

https://www.refworld.org.es/pdfid/5d7fcfefa.pdf

BBC News Mundo. 2019. «Nos enfrentamos a la amenaza muy real de una pandemia fulminante»: La advertencia de la OMS sobre el riesgo de una emergencia sanitaria. BBC News Mundo. https://www.bbc.com/mundo/noticias-49745206

BBVA. 2020. Programa de acompañamiento BBVA. https://www.bbva.com.co/personas/promocion/coronavirus.h tml\#credito-personal

CEPAL-FAO. 2020. Cómo evitar que la crisis del COVID-19 se transforme en una crisis alimentaria: Acciones urgentes contra el hambre en América Latina y el Caribe. CEPAL. https://repositorio.cepal.org/bitstream/handle/11362/45702/4/ S2000393_es.pdf 
Comisión Económica para América Latinal y el Caribe. 2020. El impacto de la pandemia por COVID-19 en las personas mayores. https://www.cepal.org/es/eventos/impacto-lapandemia-covid-19-personas-mayores

Comisión Interamericana de Derechos Humanos. 2019. Informe Empresas y derechos humanos: Estándares interamericanos. http://www.oas.org/es/cidh/informes/pdfs/EmpresasDDHH.p df

Comisión Interamericana de Mujeres, OEA. 2020. COVID-19 en la vida de las mujeres. Razones para reconocer los impactos diferenciados.

https://www.oas.org/es/cim/docs/ArgumentarioCOVID19ES.pdf

Contagioradio. 2020. Comunidades Wayúu acuden a la ONU para frenar la actividad del Cerrejón en medio de la pandemia. Contagio Radio.

https://www.contagioradio.com/comunidades-wayuu-acudena-la-onu-para-frenar-la-actividad-del-cerrejon-en-medio-dela-pandemia/

Deloitte. ¿Qué es el Gobierno Corporativo?Deloitte España. https://www2.deloitte.com/es/es/pages/governance-risk-andcompliance/articles/que-es-el-gobierno-corporativo.html

El Tiempo. 2020. Minutos y mensajes de texto gratis durante la cuarentena por coronavirus.

https://www.eltiempo.com/tecnosfera/novedadestecnologia/minutos-y-mensajes-de-texto-gratis-durante-lacuarentena-por-coronavirus-476112

Fundación Konrad Adenauer, Oficina Central de Alemania. 2020. Coronavirus in Lateinamerika.

https://www.kas.de/es/web/auslandsinformationen/artikel/det ail/-/content/coronavirus-in-lateinamerika

Mariana Mazzucato. 2020. La triple crisis del capitalismo. https://www.project-syndicate.org/commentary/covid19crises-of-capitalism-new-state-role-by-mariana-mazzucato2020-03/spanish?barrier=accesspaylog 
Miningwatch Canadá. 2020. Al ritmo del COVID-19, la minería ataca pueblos íntegros e indígenas.

https://miningwatch.ca/node/10452

Oficina del Alto Comisionado de las Naciones Unidas para los Derechos Humanos. 2011. Principios Rectores Sobre las Empresas y los Derechos Humanos: Puesta en Práctica del marco de las Naciones Unidas para "proteger, Respetary Remediar". United Nations. https://doi.org/10.18356/3b7fe68bes

Organización de las Naciones Unidas. 2020a. Declaración del Grupo de Trabajo de las Naciones Unidas sobre las Empresas y los Derechos Humanos. Asegurarse de que las empresas respeten los derechos humanos durante la crisis de Covid-19 y después: La relevancia de los Principios Rectores de las Naciones Unidas sobre las Empresas y los Derechos Humanos.

https://www.ohchr.org/SP/NewsEvents/Pages/DisplayNews.as px?NewsID $=25837 \&$ LangID $=S$

Organización de las Naciones Unidas. 2020b. La OMS necesita 31.000 millones de dólares para pruebas y vacunas contra el

coronavirus. https://news.un.org/es/story/2020/06/1476672

Organización de Naciones Unidas. 2020c. Responsabilidad compartida, solidaridad global: Respondiendo a los impactos socioeconómicos del COVID-19.

https://unsdg.un.org/resources/shared-responILITY-globalsolidarity-responding-socio-economic-impacts-\%20COVID-19

Organización de las Naciones Unidas para la Alimentación y la Agricultura (FAO) 2020. Salud y seguridad de los pueblos indígenas durante el Coronavirus (COVID-19), Recomendación 11. http://www.fao.org/indigenous-peoples/covid-19/es/

Organización Internacional del Trabajo. 1981. Convenio C155Convenio sobre seguridad y salud de los trabajadores. https://www.ilo.org/dyn/normlex/es/f?p=NORMLEXPUB:12100 :0::NO::P12100_ILO_CODE:C155

Organización Internacional del Trabajo. 2020. СОИD-19 and the world of work: Impact and policy responses. https://www.ilo.org/wcmsp5/groups/public/---dgreports/--dcomm/documents/briefingnote/wcms_738753.pdf 
Organización Internacional del Trabajo, Organización para la Cooperación y el Desarrollo Económicos, Oficina del Alto Comisionado de las Naciones Unidas para los Derechos Humanos, Comisión Interamericana de Derechos Humanos, UNICEF, \& UN Global Impact. 2020. Uniendo fuerzas en América Latina y el Caribe para ayudar a minimizar la crisis del Coronavirus (COVD-19) y fomentar empresas responsables y sostenibles.

https://www.ohchr.org/Documents/Issues/Business/WG/Joint CoronavirusStatement_es.pdf

Organización Mundial de la Salud. 2020. Alocución de apertura del Director General de la OMS en la rueda de prensa sobre la COVID-19 celebrada el 11 de marzo de 2020. https://www.who.int/es/dg/speeches/detail/who-directorgeneral-s-opening-remarks-at-the-media-briefing-on-covid-19--11-march-2020

Organización para la Cooperación y el Desarrollo Económicos. 2020a. Afrontar el Coronavirus (Covid-19) unidos en un esfuerzo global. Del confinamiento a la recuperación Respuestas medioambientales a la pandemia del COVID-19, https://read.oecd-ilibrary.org/view/?ref=130_130666agioosng94\&title=De-la-

Organización para la Cooperación y el Desarrollo Económicos. 2020b. EI COVID-19 y la Conducta Empresarial Responsable. https://read.oecd-ilibrary.org/view/?ref=130_130612yej4uhtct8\&title=El-COVID-19-y-la-Conducta-EmpresarialResponsable

Organización para la Cooperación y el Desarrollo Económicos. 2020c. Women at the core of the fight against COVID-19 crisis. https://read.oecd-ilibrary.org/view/?ref=127_127000awfnqj80me\&title=Women-at-the-core-of-the-fight-againstCOVID-19-crisis.

Pacto Global, Red Colombia. 2020. Las empresas y el cuidado de la niñez durante la pandemia: Riesgos y oportunidades. http://ideaspaz.org/especiales/infografias/guias.html

Pacto Mundial, Red Española. 2016. El sector privado ante los ODS, guía práctica para la acción. https://www.pactomundial.org/wpcontent/uploads/2016/09/Guia_ODS_online.pdf 
Publimetro. 2020. El ejemplar gesto de pastelería San Antonio ante cuarentena. Publimetro Test. https://www.publimetro.pe/nacional/2020/03/16/coronavirusel-ejemplar-gesto-de-pasteleria-san-antonio-ante-cuarentenanoticia/

Transparency International. 2020. Corrupción y Coronavirus. ¿Cómo prevenir el abuso de poder durante una pandemia mundial? https://www.transparency.org/news/feature/en_tiempos_com o_este_la_transparencia_y_la_integridad_importan_mas_que_n uncTransparency

UNICEF. 2020. Impacto del Covid-19 en los niños, niñas, adolescentes y sus familias en América Latina y el Caribe. https://www.unicef.org/lac/media/10966/file/Impact-childrencovid19-lac.pdf 